\title{
Frühsommer in Hokkaido und auf den Inseln Rishiri und Rebun (Japan)
}

\author{
ELFRIEDE SCHAUER Und Roland SCHAUER
}

\begin{abstract}
Japan's Sarobetsu-Rishiri-Rebun National Park covers the plain Sarobetsu (North-Hokkaido) and the islands Rishiri and Rebun. These islands are famous for their diversity of flowers and birds. From May to September numerous, mostly Japanese, tourists travel from Wakkanai by ferry to these islands and enjoy well-guided tours by bus and on walk ways.
\end{abstract}

\section{Zusammenfassung}

Der japanische Sarobetsu-Rishiri-Rebun Nationalpark umfasst die Ebene Sarobetsu in Nord-Hokkaido sowie die Inseln Rishiri und Rebun. Diese Inseln sind für ihre Vielfalt an Pflanzen und Vögeln bekannt. Von Mai bis September sind die Inseln für viele Japaner ein beliebtes Reiseziel. Touristen kommen meist mit der Fähre von Wakkanai. Es gibt gute Möglichkeiten, das Gebiet mit dem Bus und auf Wanderwegen zu erkunden.

\section{Einleitung}

Hokkaido sowie die vorgelagerten Inseln Rishiri und Rebun an der äußersten Nordwest-Küste Japans sind ein lohnenswertes, europäischen Naturfreunden aber wenig bekanntes Reiseziel, das hier kurz vorgestellt werden soll. Die beiden kleinen Inseln liegen an der Grenze zwischen dem Japanischen und dem Ochotskischen Meer, gegenüber der Südspitze der russischen Insel Sachalin. Eine Reise dorthin ist sehr bequem, da die touristische Infrastruktur mit bestens ausgestatteten Hotels, Restaurants und gut ausgebauten Verkehrs- und Wanderwegen ideal ist.

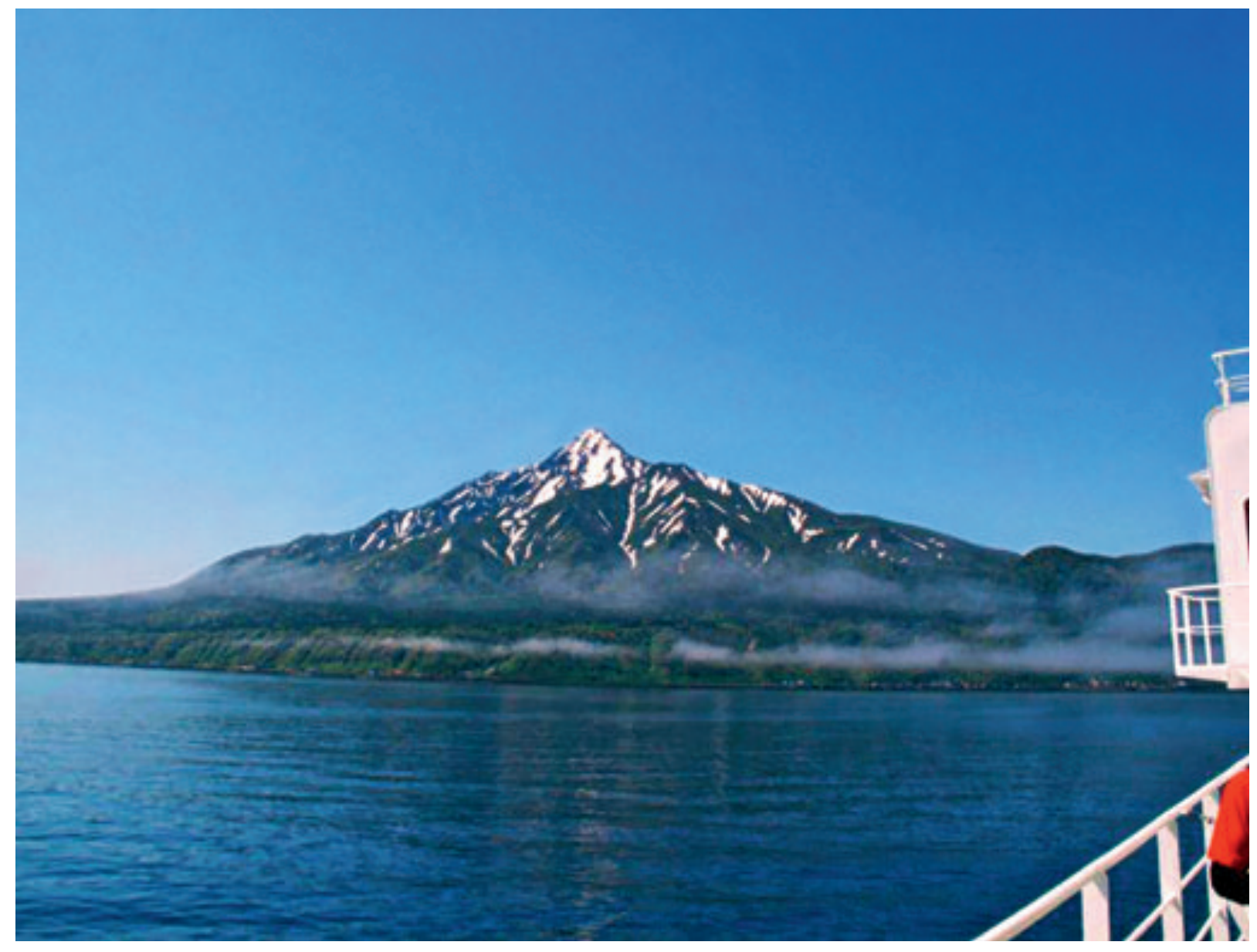




\section{Reise nach Nord-Japan}

Die Reise führt zunächst von Tokyo nach Sapporo. Dort ist zur Einstimmung auf die japanische Flora ein Besuch des Botanischen Gartens empfehlenswert. Dieser wurde im Jahr 1877 auf Anregung des Amerikaners William SMith Clark, damals Vizepräsident des Sapporo Agricultural College, angelegt. Der Garten liegt in einer fruchtbaren Ebene zwischen den Flüssen Tyohohira und Ishikari und ist der zweitälteste botanische Garten des Landes. Im Garten finden sich heimische Stauden sowie Bäume aus aller Welt. In diesem Garten wird die für Japan typische Verbundenheit der Menschen mit speziellen Pflanzen deutlich: Diverse Regionen, Städte oder Dörfer haben für sich eine Pflanze oder ein Tier ausgewählt. So ist z. B. Rosa rugosa die Blume Hokkaidos. Sapporo schmückt sich mit Flieder, der in vielen asiatischen und europäischen Sorten sowohl im botanischen Garten kultiviert wird als auch überall in der Stadt blüht und duftet.

\section{Wakkanai (Nord-Hokkaido)}

Mit dem Zug ist nach 5 Stunden Fahrt Wakkanai, die nördlichste Stadt Japans (45', 24', 14" Nord, $127^{\circ}, 48^{\prime}, 3^{\prime \prime}$ Ost), zu erreichen. Hier blühen zu Beginn des Sommers gleichzeitig Tulpen, Azaleen, Kirschen, Magnolien und einige Frühsommerblumen. Manche Bäume werden hier erst Anfang Juni grün. An der NordwestKüste von Hokkaido bildet die Sarobetsu-Ebene zusammen mit den Inseln Rishiri und Rebun den Rishiri-Rebun-Sarobetsu-Nationalpark, dessen Einrichtungen für einen nachhaltigen Schutz vorbildlich sind. In diesem Wanderparadies werden die zahlreichen Sommer-Besucher fast überall auf Holzplanken oder abgezäunten Wegen durch die Natur geführt.

\section{Rishiri (Rishiri tô) und Rebun (Rebun tô)}

Rishiri und Rebun sind für den Reichtum ihrer (Berg-)Flora und der Vogelwelt bekannt. Wäh-

Abb. 1 (S. 130): Rishiri-Fuji, vom Meer aus gesehen.

Abb. 2 (oben): Akelei (Aquilegia flabellata var. pumila f. konoi). Abb. 3 (unten): Trillium camtschaticum.
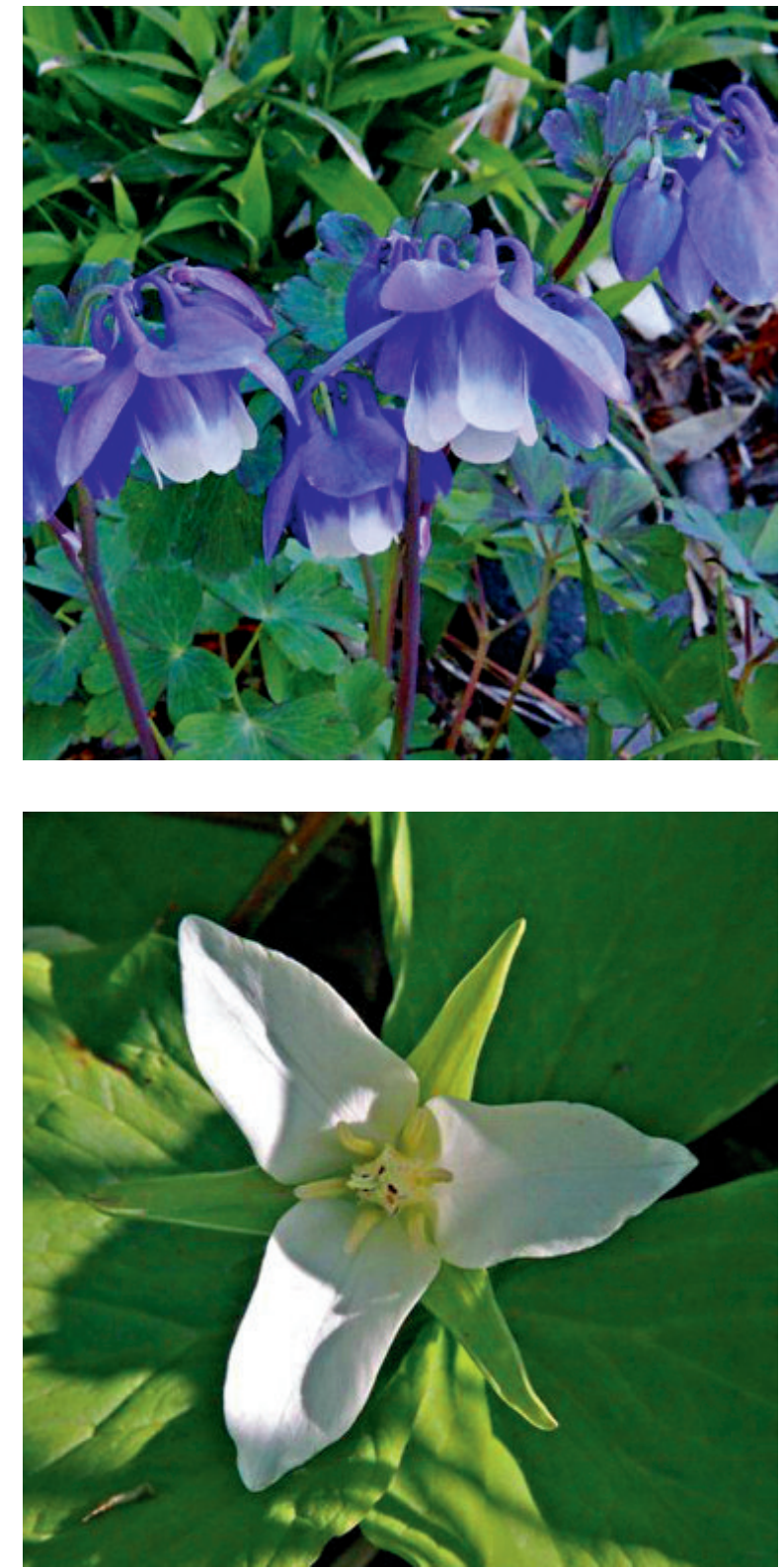

rend Rishiri eine junge Insel rein vulkanischen Ursprungs ist und saure Böden aufweist, besteht Rebun als Rest des eurasischen Kontinents außer aus Lavagestein auch aus kalkhaltigen Sedimentgesteinen. Ein $1721 \mathrm{~m}$ hoher, erloschener Vulkan beherrscht die Insel Rishiri. Da er oft auf einer Wolkenbank zu schweben scheint, wird er "floating mountain" genannt. Da der Vulkan die fast kreisrunde Insel dominiert, scheint die ganze Insel zu schwimmen (jap. ukishima = schwimmende Insel, Abb. 1). Der am Gipfel schneebedeckte Vulkan wird mit dem Fuji verglichen und deshalb auch Rishiri-Fuji genannt. Der höchste Berg auf Rebun erreicht nur 490 m Höhe.Viele Pflanzen und Tiere sind auf Hokkaido bzw. den 

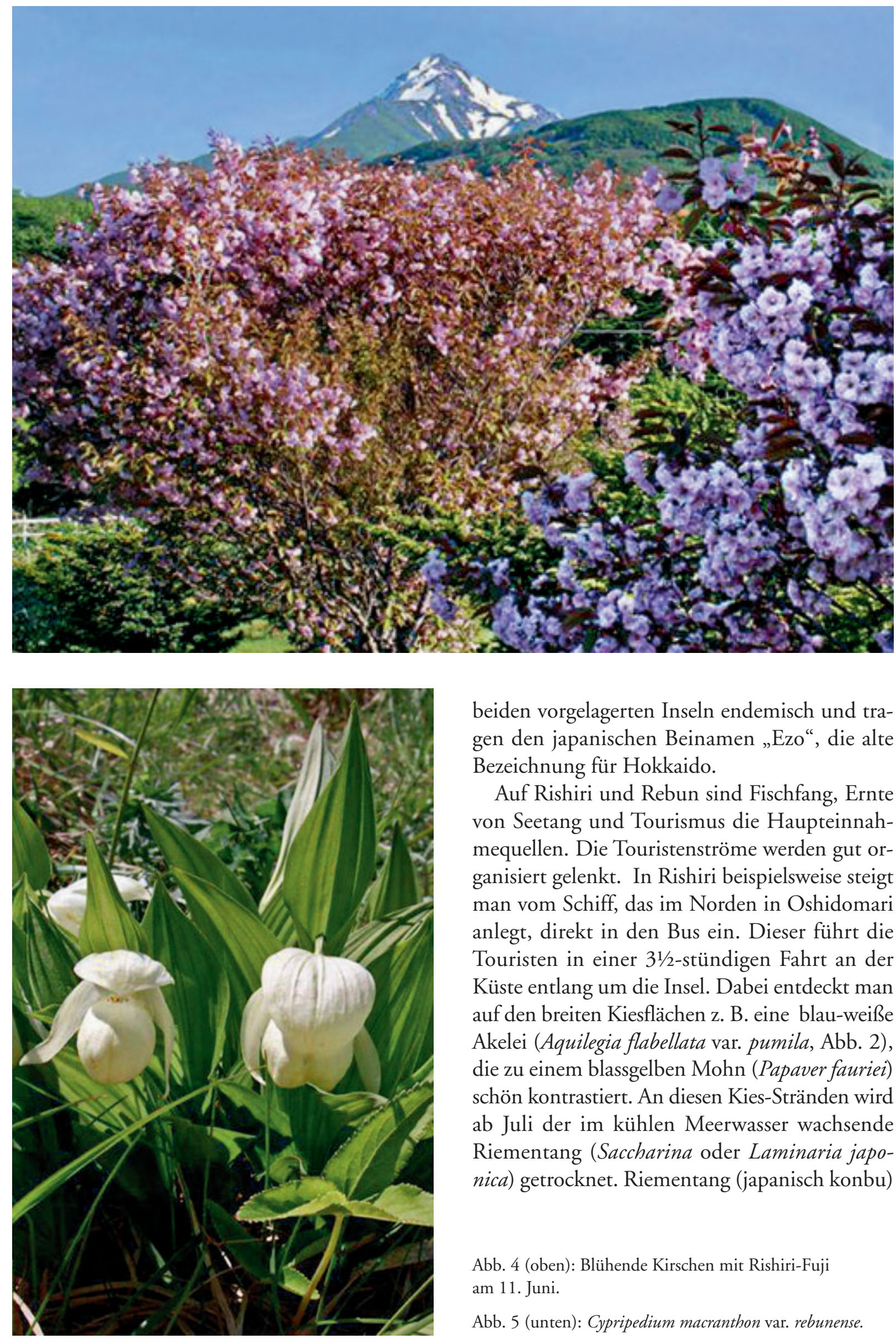

beiden vorgelagerten Inseln endemisch und tragen den japanischen Beinamen „Ezo“, die alte Bezeichnung für Hokkaido.

Auf Rishiri und Rebun sind Fischfang, Ernte von Seetang und Tourismus die Haupteinnahmequellen. Die Touristenströme werden gut organisiert gelenkt. In Rishiri beispielsweise steigt man vom Schiff, das im Norden in Oshidomari anlegt, direkt in den Bus ein. Dieser führt die Touristen in einer 31/2-stündigen Fahrt an der Küste entlang um die Insel. Dabei entdeckt man auf den breiten Kiesflächen z. B. eine blau-weiße Akelei (Aquilegia flabellata var. pumila, Abb. 2), die zu einem blassgelben Mohn (Papaver fauriei) schön kontrastiert. An diesen Kies-Stränden wird ab Juli der im kühlen Meerwasser wachsende Riementang (Saccharina oder Laminaria japonica) getrocknet. Riementang (japanisch konbu)

Abb. 4 (oben): Blühende Kirschen mit Rishiri-Fuji am 11. Juni.

Abb. 5 (unten): Cypripedium macranthon var. rebunense. 
gilt als Spezialität von Rishiri; er wird frisch verzehrt oder dient in getrockneter Form als Grundlage für Suppen, Süßigkeiten, Salate und Likör. Er ist reich an Mineralien und Glutamat. In den Wäldern erscheinen im Frühsommer die weißen Blüten einer Dreizipfel-Lilie (Trillium camtschaticum, Abb. 3), in den Wiesen blüht eine hellgelbe Trollblume (Trollius pulcher) und an feuchteren Stellen gedeiht der auch bei uns heimische Gilbweiderich (Lysimachia thyrsiflora). Die Kirschblüte ist in vollem Gang und zaubert schöne Spätnachmittags-Stimmungen (Abb. 4).

In nur 40 Minuten erreicht die Fähre von Rishiri den Hafenort Kafuka an der Südost-Ecke auf Rebun. Ein streng geschützer Endemit dieser Insel ist ein nach Zimt und Vanille duftender weißer Frauenschuh (Cypripedium macranthon var. rebunense, Abb. 5). Seine natürlichen Standorte werden streng bewacht. Das rot blühende Cypripedium macranthon ist in einem Gürtel von Moskau über Sibirien bis Nord-Japan verbreitet. In den Sumpfwiesen sind die Riesenblätter von Lysichiton camtschatcense zu entdecken. Dieses Aronstabgewächs, das auch in Mitteleuropa als Zierpflanze kultiviert wird, entwickelt im April seine Blütenstände mit weißen Hochblättern. Die Hänge der unbewaldeten Hügel sind teilweise mit einem niederen Bambusgras (jap. sasa) bedeckt. Hier blühen im Frühsommer Jakobsleiter (Polemonium caeruleum), Narzissenblütige Anemone (Anemone narcissiflora var. nipponica, Abb. 7), Trollblume (Trollius ledebouri), Weißer Germer (Veratrum album), Knabenkraut (Dactylorhiza aristata, Abb. 8), Händelwurz (Gymnadenia camtschatica, Abb. 9), Mehl-Primel (Primula modesta), diverse Läusekräuter (Pedicularis) und Storchschnabel (Geranium erianthum). Eine weitere Besonderheit im Norden ist eine schwarz-violett blühende Schachbrettblume (Fritillaria camtschatcensis, Abb. 10), die in verwilderten Gärten sowie am Rand von Gebüschen anzutreffen ist und von

Abb. 6 (oben): Hemerocallis dumontiei var. esculenta.

Abb. 7 (Mitte): Narzissenblütige Anemone (Anemone narcissiflora var. nipponica).

Abb. 8 (unten): Dactylorhiza aristata.
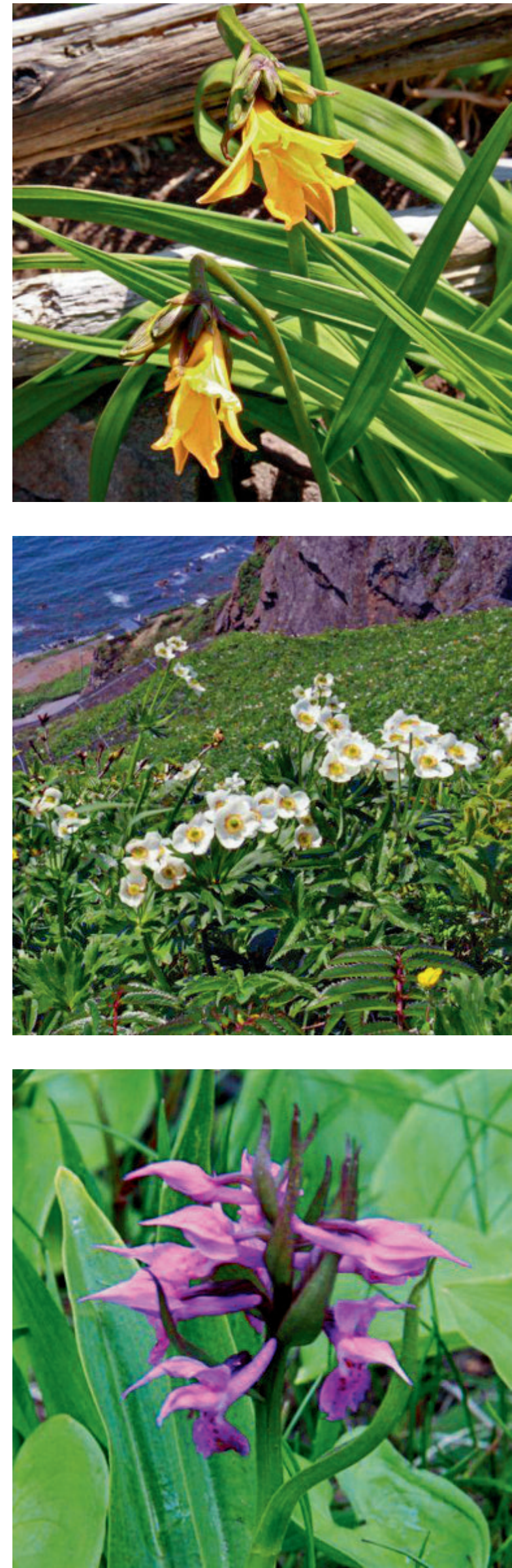

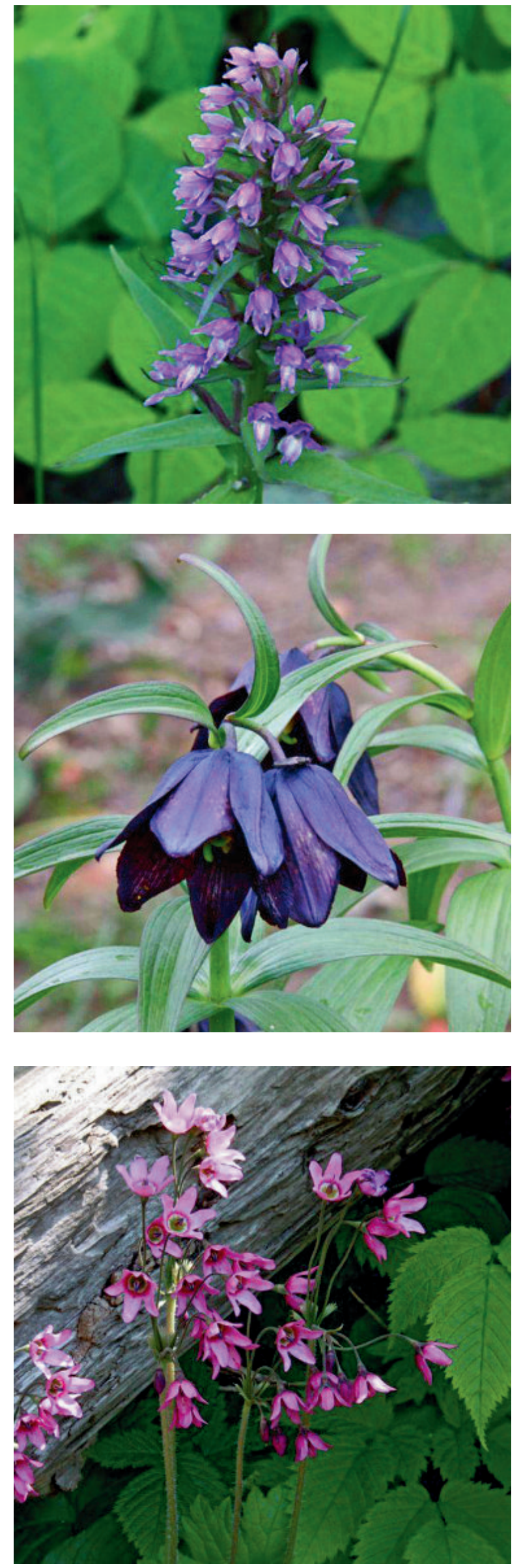

den Japanern „schwarze Lilie“ genannt wird. Sie wächst dort zusammen mit dem japanischen Heilglöckchen (Cortusa matthioli ssp. pekinensis var. sachalinensis, Abb. 11).

Mit fortschreitendem Sommer sind die Hänge dann später mit gelben Taglilien (Hemerocallis dumontiei var. esculenta, Abb. 6, S. 133), Rosa rugosa, Knöterich (Polygonum bistorta var. japonica), Japanischer Katzenminze (Nepeta subsessilis) und Ligularie (Ligularia hodgsonii) bedeckt. Berühmt ist Rebun auch wegen seiner fast bis zum Meeresstrand herabreichenden Bestände von im Juli blühendem Edelweiß (Leontopodium discolor). Ein Besuch dieser Insel ist deshalb auch im Juli und August sehr lohnenswert.

Trotz der landschaftlichen Schönheit wandern immer mehr Menschen von den Inseln ab, denn auf die kurzen warmen Sommer folgen durch sibirische Kälte beeinflusste, lange Winter, und nicht jeder junge Japaner möchte als Fischer oder Gästebetreuer arbeiten. Andererseits zieht es gerade naturliebende, wanderfreudige junge Menschen auf diese Inseln, zumindest als Touristen.

\section{Dank}

Dank gilt Prof. Teruo und Junko Yoshino, Tokyo, für das freundliche Überlassen der Abbildungen.

Abb. 9 (oben): Gymnadenia camtschatica.

Abb. 10 (Mitte): Fritillaria camtschatcensis.

Abb. 11 (unten): Cortusa matthioli ssp. pekinensis var. sachalinensis. 